ON THE OPTIMALITY OF CONSERVATION RESULTS FOR LOCAL REFLECTION IN ARITHMETIC

Author(s): A. CORDÓN-FRANCO, A. FERNÁNDEZ-MARGARIT and F. F. LARA-MARTÍN

Source: The Journal of Symbolic Logic, DECEMBER 2013, Vol. 78, No. 4 (DECEMBER 2013), pp. 1025-1035

Published by: Association for Symbolic Logic

Stable URL: https://www.jstor.org/stable/43303696

JSTOR is a not-for-profit service that helps scholars, researchers, and students discover, use, and build upon a wide range of content in a trusted digital archive. We use information technology and tools to increase productivity and facilitate new forms of scholarship. For more information about JSTOR, please contact support@jstor.org.

Your use of the JSTOR archive indicates your acceptance of the Terms \& Conditions of Use, available at https://about.jstor.org/terms 


\title{
ON THE OPTIMALITY OF CONSERVATION RESULTS FOR LOCAL REFLECTION IN ARITHMETIC
}

\author{
A. CORDÓN-FRANCO, A. FERNÁNDEZ-MARGARIT, AND F. F. LARA-MARTÍN
}

\begin{abstract}
Let $T$ be a recursively enumerable theory extending Elementary Arithmetic EA. L. D. Beklemishev proved that the $\Sigma_{2}$ local reflection principle for $T . \operatorname{Rfn}_{\Sigma_{2}}(T)$, is conservative over the $\Sigma_{1}$ local reflection principle, $\operatorname{Rfn}_{\Sigma_{1}}(T)$, with respect to boolean combinations of $\Sigma_{1}$-sentences: and asked whether this result is best possible. In this work we answer Beklemishev's question by showing that $\Pi_{2}$-sentences are not conserved for $T=\mathrm{EA}+$ " $f$ is total." where $f$ is any nondecreasing computable function with elementary graph. We also discuss how this result generalizes to $n>0$ and obtain as an application that for $n>0, I \Pi_{n+1}^{-}$is conservative over $I \Sigma_{n}$ with respect to $\Pi_{n+2}$-sentences.
\end{abstract}

§1. Introduction. This work was motivated by a question of L. D. Beklemishev on the optimality of a conservation result for reflection principles in first order arithmetic. Reflection principles for a given theory $T$ are axiom schemes expressing the soundness of $T$. More precisely, if $T$ is a recursively enumerable (r.e.) arithmetic theory extending Elementary Arithmetic EA and $\square_{T}(x)=\exists y \operatorname{Prf}_{T}(x, y)$ denotes a standard provability predicate for $T$, the local reflection principle for $T$ is the axiom scheme given by

$$
\operatorname{Rfn}(T): \square_{T}(\ulcorner\varphi\urcorner) \rightarrow \varphi,
$$

where $\varphi$ ranges over all sentences of the language of $T$ and $\ulcorner\varphi\urcorner$ denotes (the numeral of) the Gödel number of $\varphi$. The term local refers to the fact that the scheme is restricted to sentences, in contrast with the uniform reflection principle for $T$, where formulas with free variables are allowed:

$$
\operatorname{RFN}(T): \square_{T}(\ulcorner\varphi(\dot{x})\urcorner) \rightarrow \varphi(x) .
$$

In [3], using provability logic techniques, Beklemishev showed that over $T$, full local reflection $\operatorname{Rfn}(T)$ is $\Gamma$-conservative over local reflection restricted to $\Gamma$-sentences, denoted $\operatorname{Rfn}_{\Gamma}(T)$, for $\Gamma=\Sigma_{n}$ or $\Pi_{n}$. More precisely,

THEOREM 1.1 (Beklemishev, [3]). 1. For $n>1, T+\operatorname{Rfn}(T)$ is conservative over $T+\operatorname{Rfn}_{\Sigma_{n}}(T)$ with respect to $\Sigma_{n}$-sentences (and dually for $\Pi_{n}$ ).

2. $T+\operatorname{Rfn}(T)$ is conservative over $T+\operatorname{Rfn}_{\Sigma_{1}}(T)$ with respect to $\mathscr{B}\left(\Sigma_{1}\right)(=$ boolean combinations of $\left.\Sigma_{1}\right)$ sentences.

Received September 12, 2011.

This work was supported by grant MTM2008-06435, Ministerio de Ciencia e Innovación, Spain. Co-financed with FEDER funds, EU. 
In [3] Beklemishev noted that part (1) in the above theorem is best possible with respect to arithmetic complexity and in [6] he raised the problem of determining the optimality of part (2). In general, $T+\operatorname{Rfn}(T)$ is neither $\Pi_{2}$ nor $\Sigma_{2}$ conservative over $T+\operatorname{Rfn}_{\Sigma_{1}}(T)$, for otherwise $T+\operatorname{Rfn}_{\Pi_{2}}(T)\left(\right.$ resp. $\left.T+\operatorname{Rfn}_{\Sigma_{2}}(T)\right)$ would collapse to $T+\operatorname{Rfn}_{\Sigma_{1}}(T)$ and it is known that the local reflection hierarchy is proper unless its $\Sigma_{1}$ level is already inconsistent. Nevertheless, it follows from part (2) that both $T+\operatorname{Rfn}_{\Pi_{2}}(T)$ and $T+\operatorname{Rfn}_{\Sigma_{2}}(T)$ are $\mathscr{B}\left(\Sigma_{1}\right)$-conservative over $T+\operatorname{Rfn}_{\Sigma_{1}}(T)$ and now there could be room for improvement. In fact, by Proposition 4.5 in [5] conservativity of $T+\operatorname{Rfn}_{\Pi_{2}}(T)$ over $T+\operatorname{Rfn}_{\Sigma_{1}}(T)$ can be extended to $\Sigma_{2}$-sentences if $T \subseteq \Pi_{2}$. In contrast, the corresponding question for $T+\operatorname{Rfn}_{\Sigma_{2}}(T)$ seems to have been open.

QUESTION 1 (Beklemishev). Is $T+\operatorname{Rfn}_{\Sigma_{2}}(T)$ conservative over $T+\operatorname{Rfn}_{\Sigma_{1}}(T)$ with respect to $\Pi_{2}$-sentences?

Typically, non $\Pi_{2}$-conservation can be shown by exhibiting a computable function provably total in a theory and not in the other. However, $\operatorname{Rfn}_{\Sigma_{2}}(T)$ has quantifier complexity $\Sigma_{2}$ and so $T+\operatorname{Rfn}_{\Sigma_{2}}(T)$ and $T+\operatorname{Rfn}_{\Sigma_{1}}(T)$ share the same class of provably total functions. This motivates the following problem on general arithmetic theories, of which Beklemishev's Question 1 for $T \subseteq \mathscr{B}\left(\Sigma_{1}\right)$ is a particular case $\left(\operatorname{Th}_{\Gamma}(S)\right.$ denotes the $\Gamma$-consequences of a theory $S$ ):

Question 2. Suppose $S$ contains EA. Are $\operatorname{Th}_{\Pi_{2}}\left(\operatorname{Th}_{\Sigma_{2}}(S)\right)$ and $\operatorname{Th}_{\mathscr{B}\left(\Sigma_{1}\right)}(S)$ deductively equivalent?

In this work we solve in the negative both questions for a wide class of theories and apply the proof ideas to obtain some new results on local reflection. Our methods are model-theoretic and we exploit the connections between reflection principles and induction schemes in the spirit of Kreisel and Lévy' [13].

The paper is organized as follows. Sections 1 and 2 are introductory. Section 3 presents a preliminary result that puts in context the negative answer to Question 2. In Section 4 we show that Question 1 has the negative answer for $T=\mathrm{EA}+$ " $f$ is total," where $f$ is any nondecreasing computable function with elementary graph; and, similarly, we solve in the negative Question 2 for every consistent, r.e. extension of EA $+R f n_{\Sigma_{2}}(E A)$. Both results are derived from Theorem 4.4, which is an unboundness theorem of independent interest. In Section 5, we deal with results à la Kreisel-Lévy relating local reflection and various forms of induction. We improve known results for $\operatorname{Rfn}_{\Sigma_{2}}(T)$ and $\operatorname{Th}_{\Pi_{1}}(T+\operatorname{Rfn}(T))$ as well as we fill an obvious gap in our understanding of partial local reflection by obtaining a Kreisel and Lévy-like theorem for $\operatorname{Rfn}_{\Sigma_{1}}(T)$. Finally, in Section 6 we discuss how our results generalize to $n>0$ and obtain a new conservation theorem for parameter free induction: $I \Pi_{n+1}^{-}$is $\Pi_{n+2}$-conservative over $I \Sigma_{n}$.

§2. Basic notions and notation. Our notation is standard and we assume that the reader is familiar with the basic notions of first order arithmetic (we recommend [11] and [10] for a detailed introduction to the subject; [12] for results on parameter free schemes; and [6] for information on reflection principles). To a large extent, our results are independent of the language we are working in. Nevertheless, for the sake of definiteness we assume that we work in the language $\mathscr{L}_{\exp }=\left\{0,1,+,{ }^{\circ},<\right.$, exp $\}$ 
extending that of Peano Arithmetic $P A$ with a symbol for the function $2^{x}$. Also, we assume that all the theories we shall deal with are extensions of Elementary Arithmetic EA, which is axiomatized by a finite set of defining axioms for the symbols in $\mathscr{L}_{\text {exp }}$ plus the scheme of induction for bounded (elementary) formulas of $\mathscr{L}_{\text {exp }}$. Finally, we also assume, sometimes without explicit mention, that every theory $T$ for which we consider reflection principles is an elementary presented extension of EA; the set of its axioms being represented by an elementary formula of the form $A x_{\mathrm{EA}}(x) \vee A x_{T}(x)$.

We recall some notation on parameter free induction schemes from [12]. If $\Gamma$ is a class of formulas, the scheme $I \Gamma^{-}$is given by

$$
\varphi(0) \wedge \forall x(\varphi(x) \rightarrow \varphi(x+1)) \rightarrow \forall x \varphi(x),
$$

where $\varphi(x) \in \Gamma^{-}$(we write $\varphi(x) \in \Gamma^{-}$to mean that $\varphi$ is in $\Gamma$ and contains no other free variables than $x$ ).

We will be also concerned with a number of inference rules associated to induction principles. Given an inference rule $R$ and a theory $T, T+R$ denotes the closure of $T$ under $R$ and first order logic; while [T,R] denotes the closure of $T$ under non-nested applications of $R$ and first order logic. A rule $R_{1}$ is reducible to $R_{2}$ if $\left[T, R_{1}\right] \subseteq\left[T, R_{2}\right]$ for every theory $T$ extending EA; two rules $R_{1}$ and $R_{2}$ are congruent, written $R_{1} \cong R_{2}$, if they are mutually reducible to each other.

Finally, if $\mathfrak{A}$ and $\mathfrak{B}$ are $\mathscr{L}_{\text {exp }}$-structures we write $\mathfrak{A} \prec_{n} \mathfrak{B}$ to mean that $\mathfrak{A}$ is a $\Sigma_{n}$-elementary substructure of $\mathfrak{B}$, i.e., for all $\varphi(x) \in \Sigma_{n}$ and $a \in \mathfrak{A}, \mathfrak{A} \models \varphi(a)$ iff $\mathfrak{B} \models \varphi(a)$. Natural examples of $\Sigma_{n}$-elementary substructures are provided by submodels of $\Sigma_{n}$-definable elements, $\mathscr{K}_{n}(\mathfrak{A})$. Recall that $a$ is a $\Sigma_{n}$-definable element of a model $\mathfrak{A}$ if there is $\varphi(x) \in \Sigma_{n}$ such that $a$ is the unique element satisfying $\varphi(x)$ in $\mathfrak{A}$, and

- $\mathscr{K}_{n}(\mathfrak{A})$ denotes the set of all $\Sigma_{n}$-definable elements of $\mathfrak{A} ; \mathscr{I}_{n}(\mathfrak{A})$ denotes the initial segment of $\mathfrak{A}$ determined by $\mathscr{K}_{n}(\mathfrak{A})$.

- $\mathscr{K}_{n}^{1}(\mathfrak{A})=\mathscr{K}_{n}\left(\mathfrak{A}, \mathscr{I}_{n}(\mathfrak{A})\right)$ denotes the set of all elements of $\mathfrak{A}$ which are $\Sigma_{n^{-}}$ definable with a parameter from $\mathscr{I}_{n}(\mathfrak{A})$.

Note that for $n \geq 1$, if $\mathfrak{A} \models \mathrm{EA}+I \Sigma_{n-1}^{-}$then $\mathscr{K}_{n}(\mathfrak{A}) \prec_{n} \mathfrak{A}$, see e.g., [10].

§3. A preliminary result. At first glance the negative answer to Question 2 might seem to be rather obvious. However, it should be compared with the following result stating that a dual version of that question has a positive answer. Thus, our work also brings into evidence the different behavior of the dual classes $\operatorname{Th}_{\Pi_{2}}\left(\mathrm{Th}_{\Sigma_{2}}(S)\right)$ and $\mathrm{Th}_{\Sigma_{2}}\left(\mathrm{Th}_{\Pi_{2}}(S)\right)$ for a general arithmetic theory $S$.

Proposition 3.1. If $S$ implies EA then $\mathrm{Th}_{\Sigma_{2}}\left(\mathrm{Th}_{\Pi_{2}}(S)\right) \equiv \mathrm{Th}_{\mathscr{B}\left(\Sigma_{1}\right)}(S)$.

Proof. It is obvious that $\operatorname{Th}_{\mathscr{B}\left(\Sigma_{1}\right)}(S) \subseteq \mathrm{Th}_{\Sigma_{2}}\left(\mathrm{Th}_{\Pi_{2}}(S)\right)$. For the opposite direction, assume that $\mathfrak{A}$ is a model of $\operatorname{Th}_{\mathscr{B}\left(\Sigma_{1}\right)}(S)$. Consider the theory

$$
S^{\prime}=\mathrm{Th}_{\Sigma_{1}}(\mathfrak{A})+\mathrm{Th}_{\Pi_{1}}(\mathfrak{A})+S,
$$

where $\operatorname{Th}_{\Gamma}(\mathfrak{A})$ denotes the set of all $\Gamma$-sentences which are true in the model $\mathfrak{A}$. Clearly, $S^{\prime}$ is consistent. Let $\mathfrak{B}$ be a model of $S^{\prime}$. Then $\mathscr{K}_{1}(\mathfrak{A}) \prec_{1} \mathfrak{A}$ and $\mathscr{K}_{1}(\mathfrak{B}) \prec_{1}$ $\mathfrak{B}$; and, as a consequence, $\mathscr{K}_{1}(\mathfrak{B}) \models \mathrm{Th}_{\Pi_{2}}(S)$ and $\mathfrak{A} \models \mathrm{Th}_{\Sigma_{2}}\left(\mathscr{K}_{1}(\mathfrak{A})\right)$. But it follows from the fact that $\mathfrak{A}$ and $\mathfrak{B}$ satisfy the same $\Sigma_{1}$-sentences that $\mathscr{K}_{1}(\mathfrak{A})$ and $\mathscr{K}_{1}(\mathfrak{B})$ 
are isomorphic (if $\varphi(x)$ defines $a \in \mathfrak{A}$, map $a$ to the unique $b \in \mathfrak{B}$ satisfying $\varphi(x)$ in $\mathfrak{B})$. Consequently, $\mathfrak{A} \models \mathrm{Th}_{\Sigma_{2}}\left(\mathrm{Th}_{\Pi_{2}}(S)\right)$.

Note that the presence of the exponentiation in $S$ is irrelevant and the result easily generalizes to $\operatorname{Th}_{\Sigma_{n+2}}\left(\operatorname{Th}_{\Pi_{n+2}}(S)\right) \equiv \operatorname{Th}_{\mathscr{B}\left(\Sigma_{n+1}\right)}(S)$ if $S$ contains $I \Sigma_{n}^{-}$.

Proposition 3.1 provides us with a recipe for lifting a $\mathscr{B}\left(\Sigma_{1}\right)$-conservation result to $\Sigma_{2}$-conservation. Namely, if $T_{1}$ is a $\Pi_{2}$-theory extending $\Delta_{0}$-induction and $T_{1}$ is $\mathscr{B}\left(\Sigma_{1}\right)$-conservative over $T_{2}$, then $\Sigma_{2}$-sentences are also conserved. In particular, this gives us a simple proof of the known fact that the dual version of Question 1 has a positive answer for $\Pi_{2}$-theories, i.e., $T+\operatorname{Rfn}_{\Pi_{2}}(T)$ is $\Sigma_{2}$-conservative over $T+\operatorname{Rfn}_{\Sigma_{1}}(T)$ if $T \subseteq \Pi_{2}$.

Despite its simplicity it seems that Proposition 3.1 has not been widely known, although some cases of theories that turn out to have axiomatizations of unexpectedly low quantifier complexity have been observed in the literature. For example, in [12] it is proved that, for $n>0$, the $\mathscr{B}\left(\Sigma_{n+1}\right)$-consequences of $I \Sigma_{n}$ axiomatize the $\Sigma_{n+2}$-consequences of $I \Sigma_{n}$. Also, in [5] it is shown that over $T$, the $\Sigma_{2}$-consequences of $\operatorname{RFN}_{\Sigma_{1}}(T)$ (uniform reflection restricted to $\Sigma_{1}$ formulas) can be axiomatized by $\operatorname{Rfn}_{\Sigma_{1}}(T) \subseteq \mathscr{B}\left(\Sigma_{1}\right)$. Both results can be seen as particular cases of Proposition 3.1.

§4. An unboundedness theorem for $\mathrm{Th}_{\Pi_{2}}\left(T+\mathrm{Rf}_{\Sigma_{2}}(T)\right)$. The so-called unboundedness theorems due to Kreisel and Lévy [13] state that $\operatorname{Rfn}_{\Pi_{n}}(T)$ is not contained in any consistent finite extension of $T$ of complexity $\Sigma_{n}$ (and dually for $\operatorname{Rfn}_{\Sigma_{n}}(T)$ ). Here we obtain a variant of these results for the $\Pi_{2}$-consequences of $T+\operatorname{Rfn}_{\Sigma_{2}}(T)$. To this end, a crucial fact is that, somewhat surprisingly, $\operatorname{Rfn}_{\Sigma_{2}}(T)$ allows for a modicum of parametric $\Sigma_{1}$-induction. In [8], we proved that $I \Pi_{n}^{-}$is equivalent to the following local variants of the $\Sigma_{n}$-induction scheme, where the conclusion of the induction axiom is relativized to definable elements:

- The scheme $I\left(\Sigma_{n}^{-}, \mathscr{K}_{n}\right)$ is given by

$$
\varphi(0) \wedge \forall x(\varphi(x) \rightarrow \varphi(x+1)) \rightarrow \forall x \in \mathscr{K}_{n} \varphi(x)
$$

where $\varphi(x) \in \Sigma_{n}^{-}$and a quantifier of the form $\forall x \in \mathscr{K}_{n}$ in front of a formula $\Psi(x)$ is intended as a shorthand for the scheme:

$$
\left\{\forall x\left[\operatorname{Def}_{\delta}(x) \rightarrow \Psi(x)\right]: \delta \in \Sigma_{n}\right\}
$$

(we write $\operatorname{Def}_{\delta}(x, v)$ to denote $\delta(x, v) \wedge \forall x, x^{\prime}\left(\delta(x, v) \wedge \delta\left(x^{\prime}, v\right) \rightarrow x=x^{\prime}\right)$, for a formula $\delta(x, v)$, or $\operatorname{Def}_{\delta}(x)$ if $\delta(x)$ does not contain any parameters).

- The scheme $I\left(\Sigma_{n}, \mathscr{I}_{n}, \mathscr{K}_{n}^{1}\right)$ is given by:

$$
\forall v \in \mathscr{K}_{n}^{1}\left[\varphi(0, v) \wedge \forall x(\varphi(x, v) \rightarrow \varphi(x+1, v)) \rightarrow \forall x \in \mathscr{I}_{n} \varphi(x, v)\right],
$$

where $\varphi(x, v) \in \Sigma_{n}$, a quantifier $\forall x \in \mathscr{I}_{n} \Psi(x)$ unravels to

$$
\left\{\forall x, y\left[\operatorname{Def}_{\dot{\delta}}(y) \wedge x \leq y \rightarrow \Psi(x)\right]: \delta \in \Sigma_{n}\right\},
$$

and a quantifier of the form $\forall x \in \mathscr{K}_{n}^{1} \Psi(x)$ unravels to

$$
\left\{\forall x, y, z\left[\operatorname{Def}_{\delta_{1}}(z) \wedge y \leq z \wedge \operatorname{Def}_{\delta_{2}}(x, y) \rightarrow \Psi(x)\right]: \delta_{1}, \delta_{2} \in \Sigma_{n}\right\} .
$$

The equivalence between $I \Pi_{n}^{-}$and the above schemes was essentially proved in [8], see (the proofs of) Proposition 2.1 and Theorem 2.3 there.

PROPOSITION 4.1. Over EA $+I \Sigma_{n-1}^{-}, I \Pi_{n}^{-} \equiv I\left(\Sigma_{n}^{-}, \mathscr{K}_{n}\right) \equiv I\left(\Sigma_{n}, \mathscr{I}_{n}, \mathscr{K}_{n}^{1}\right)$. 
Since $T+\operatorname{Rfn}_{\Sigma_{2}}(T)$ contains $I \Pi_{1}^{-}$by Theorem 1 of [4], it follows that

Proposition 4.2. $T+\operatorname{Rfn}_{\Sigma_{2}}(T)$ implies $I\left(\Sigma_{1}, \mathscr{I}_{1}, \mathscr{K}_{1}^{1}\right)$.

Equipped with $I\left(\Sigma_{1}, \mathscr{I}_{1}, \mathscr{K}_{1}^{1}\right)$ a proof of the desired unboundness theorem proceeds as follows. Suppose $T=\mathrm{EA}+$ " $f$ is total," where $f$ is a computable function. Then a natural candidate for "a hardest $\Pi_{2}$-problem" for the theory $T+\operatorname{Rfn}_{\Sigma_{2}}(T)$ is: $\forall x \in \mathscr{I}_{1} \forall z \in \mathscr{K}_{1}^{1}$ " $f^{x}(z)$ exists," where $f^{x}$ is the $x$-th iterate of $f$. In what follows, we shall show that this is actually the case whenever the graph of $f$ is defined by an EA-honest formula:

Definition 4.3. We say that a formula $y=f(x)$ is EA-honest if

1. $y=f(x)$ is elementary,

2. EA $\vdash y=f(x) \rightarrow y \geq 2^{x}$, and

3. $\mathrm{EA} \vdash x_{1} \leq x_{2} \wedge y_{1}=f\left(x_{1}\right) \wedge y_{2}=f\left(x_{2}\right) \rightarrow y_{1} \leq y_{2}$.

By Proposition 5.4 of [2] a theory $T$ can be written as EA + " $f$ is total," where $y=f(x)$ is EA-honest, if and only if $T$ is a finite $\Pi_{2}$-extension of EA closed under the $\Sigma_{1}$-collection rule

$$
\Sigma_{1} \text {-CR: } \frac{\forall x \exists y \varphi(x, y)}{\forall z \exists u \forall x \leq z \exists y \leq u \varphi(x, y)},
$$

where $\varphi(x, y) \in \Sigma_{1}$. In addition, if $y=f(x)$ is an EA-honest formula then, using elementary coding of sequences, the iteration of $f$ can be expressed by the following elementary formula (denoted by $y=f^{x}(z)$ ):

$$
\exists s \leq b t\left[\text { length }(s)=x+1 \wedge(s)_{0}=z \wedge \forall i<x\left((s)_{i+1}=f\left((s)_{i}\right)\right) \wedge y=(s)_{x}\right],
$$

where $b t$ is a bounding term for the code of a sequence consisting of $(x+1)$-many $y$ 's. We are now in a position to state the main result of this section:

THEOREM 4.4 (Unboundedness). Suppose $T$ is a finite $\Pi_{2}$-extension of EA closed under $\Sigma_{1}-C R$. Then, $\mathrm{Th}_{\Pi_{2}}\left(T+\mathrm{Rfn}_{\Sigma_{2}}(T)\right)$ is not contained in any consistent, r.e. extension of $T$ by $\mathscr{B}\left(\Sigma_{1}\right)$-sentences.

Proof. Put $T \equiv \mathrm{EA}+\forall x \exists ! y(y=f(x))$, where $y=f(x)$ is EA-honest. Let $\Gamma$ be an r.e. set of $\mathscr{B}\left(\Sigma_{1}\right)$-sentences satisfying that $T+\Gamma$ is consistent. We shall construct a model of $T+\Gamma$ in which $T h_{\Pi_{2}}\left(T+\operatorname{Rfn}_{\Sigma_{2}}(T)\right)$ fails. First of all, note that $T+\Gamma$ does not imply the set of all true $\Pi_{1}$ sentences $T_{\Pi_{1}}(\mathbb{N})$, for otherwise it would follow that $T h_{\Pi_{1}}(T+\Gamma)=T h_{\Pi_{1}}(\mathbb{N})$ and this is impossible since the first set is r.e. and the second one is $\Pi_{1}^{0}$-complete. Thus, there is $\mathfrak{A} \models T+\Gamma$ in which $\operatorname{Th}_{\Pi_{1}}(\mathbb{N})$ fails and so $\mathscr{K}_{1}(\mathfrak{A})$ is nonstandard. By considering an elementary extension of $\mathfrak{A}$ if necessary, we may also assume that $\mathscr{K}_{1}(\mathfrak{A})$ is bounded above in $\mathfrak{A}$. Then, it holds that $\mathscr{K}_{1}^{1}(\mathfrak{A}) \neq \mathscr{I}_{1}(\mathfrak{A})$, for otherwise $\mathscr{I}_{1}(\mathfrak{A})$ would have a proper $\Sigma_{1}$-elementary end extension and hence $\mathscr{I}_{1}(\mathfrak{A}) \models B \Sigma_{2}$ by Theorem B of [16]. But $I \Sigma_{1} \subseteq B \Sigma_{2}$ and $I \Sigma_{1}$ is well-known to fail in $\mathscr{I}_{1}(\mathfrak{A})$ whenever $\mathscr{I}_{1}(\mathfrak{A})$ is nonstandard. It thus follows that there is $a \in \mathscr{K}_{1}^{1}(\mathfrak{A})$ such that $\mathscr{I}_{1}(\mathfrak{A})<a$. Consider $\varphi(x, y, v)$ elementary and $b \in \mathscr{I}_{1}(\mathfrak{A})$ such that $\exists y \varphi(x, y, b)$ defines $a$ in $\mathfrak{A}$. Since $\varphi$ is bounded, there is a minimal $c$ satisfying $\varphi\left((z)_{0},(z)_{1}, b\right)$ in $\mathfrak{A}$. It is clear that $(c)_{0}=a$ and so $\mathscr{I}_{1}(\mathfrak{A})<a<c$. Now define $\mathfrak{B}$ to be initial segment of $\mathfrak{A}$ determined by the standard iterations $f^{k}(c)$, i.e.,

$$
\mathfrak{B}=\left\{d \in \mathfrak{A}: \exists k \in \omega, \mathfrak{A} \models \exists y\left(y=f^{k}(c) \wedge d \leq y\right)\right\} .
$$


It follows that $\mathfrak{B}$ is an initial substructure of $\mathfrak{A}, \mathfrak{B} \prec_{0} \mathfrak{A}$, and $\mathscr{K}_{1}(\mathfrak{A}) \subseteq \mathfrak{B}$.

In addition, we have $\mathfrak{B} \models T+\Gamma$. Note that $\mathfrak{B} \models T$, since $y=f(x)$ defines a total function in $\mathfrak{B}$. To see $\mathfrak{B} \models \Gamma$, consider $\theta \in \Gamma$. By logical operations,

$$
\theta \equiv\left(\theta_{1}^{0} \vee \exists x \theta_{2}^{0}(x)\right) \wedge \cdots \wedge\left(\theta_{1}^{n} \vee \exists x \theta_{2}^{n}(x)\right),
$$

with $\theta_{1}^{i} \in \Pi_{1}$ and $\theta_{2}^{i}(x) \in \Delta_{0}$. Fix $i \leq n$. Since $\mathfrak{A}$ satisfies $\Gamma, \mathfrak{A} \models \theta_{1}^{i} \vee \exists x \theta_{2}^{i}(x)$.

Case 1: $\mathfrak{A}=\theta_{1}^{i}$. Then $\theta_{1}^{i}$ is also valid in $\mathfrak{B}$ since $\mathfrak{B} \prec_{0} \mathfrak{A}$.

Case 2: $\mathfrak{A} \models \exists x \theta_{2}^{i}(x)$. Then there is $a_{i} \in \mathscr{K}_{1}(\mathfrak{A})$ such that $\mathfrak{A} \models \theta_{2}^{i}\left(a_{i}\right)$. But $a_{i} \in \mathfrak{B}$ since $a_{i} \leq c$. So, $\mathfrak{B} \models \exists x \theta_{2}^{i}(x)$.

Hence, $\mathfrak{B} \models \theta$, as required.

Finally, we prove that $\mathfrak{B} \not=\mathrm{Th}_{\Pi_{2}}\left(T+\operatorname{Rfn}_{\Sigma_{2}}(T)\right)$.

First, let us observe that

$$
\operatorname{Th}_{\Pi_{2}}\left(T+\operatorname{Rfn}_{\Sigma_{2}}(T)\right) \vdash \forall x \in \mathscr{I}_{1} \forall z \in \mathscr{K}_{1}^{1} \exists y\left(y=f^{x}(z)\right) .
$$

Indeed, by Proposition 4.2, $T+\operatorname{Rfn}_{\Sigma_{2}}(T)$ proves $\forall x \in \mathscr{I}_{1} \forall z \in \mathscr{K}_{1}^{1} \exists y\left(y=f^{x}(z)\right)$, and a quantifier of the form $\forall x \in \mathscr{I}_{1}$ or $\forall z \in \mathscr{K}_{1}^{1}$ in front of a $\Pi_{2}$-formula unravels to a scheme of $\Pi_{2}$-formulas; hence, $(\dagger)$ follows.

Now observe that $c \in \mathscr{K}_{1}^{1}(\mathfrak{B})$. In fact, $c=(\mu z)\left(\varphi\left((z)_{0},(z)_{1}, b\right)\right)$ in $\mathfrak{B}$, with $b \in \mathscr{I}_{1}(\mathfrak{A})$. But it follows from $\mathfrak{B} \prec_{0} \mathfrak{A}$ and $\mathscr{K}_{1}(\mathfrak{A}) \subseteq \mathfrak{B}$ that $\mathscr{K}_{1}(\mathfrak{B})=\mathscr{K}_{1}(\mathfrak{A})$. So, $b \in \mathscr{I}_{1}(\mathfrak{B})$ and hence $c \in \mathscr{K}_{1}^{1}(\mathfrak{B})$.

Pick $e \in \mathscr{K}_{1}(\mathfrak{A})$ nonstandard. Since $f(x) \geq 2^{x} \geq x$, EA proves that $y=f^{x}(z)$ defines a nondecreasing function in the variable $x$. So, $f^{e}(c)$ does not exist in $\mathfrak{B}$. Consequently, $\operatorname{Th}_{\Pi_{2}}\left(T+\operatorname{Rfn}_{\Sigma_{2}}(T)\right)$ fails in $\mathfrak{B}$ by $(\dagger)$.

We can now derive the answers to Question 1 and 2 as direct corollaries.

Corollary 4.5 (Answer to Question 1). Suppose $T_{1}$ is a finite $\Pi_{2}$-extension of EA closed under $\Sigma_{1}-C R, T_{2}$ is an r.e. set of $\mathscr{B}\left(\Sigma_{1}\right)$-sentences and $T=T_{1}+T_{2}$. Then $T+\operatorname{Rfn}_{\Sigma_{2}}(T)$ is not $\Pi_{2}$-conservative over $T+\operatorname{Rfn}_{\Sigma_{1}}(T)$ provided $T+\operatorname{Rfn}_{\Sigma_{1}}(T)$ is consistent.

Proof. It follows from Theorem 4.4 for $T_{1}$, because $T+\operatorname{Rfn}_{\Sigma_{1}}(T)$ is a consistent, r.e. extension of $T_{1}$ by $\mathscr{B}\left(\Sigma_{1}\right)$-sentences.

Corollary 4.6 (Answer to Question 2). Suppose $S$ is a consistent, r.e. extension of $\mathrm{EA}+\mathrm{Rfn}_{\Sigma_{2}}(\mathrm{EA})$. Then $\mathrm{Th}_{\Pi_{2}}\left(\mathrm{Th}_{\Sigma_{2}}(S)\right)$ is strictly stronger than $\mathrm{Th}_{\mathscr{B}\left(\Sigma_{1}\right)}(S)$.

Proof. Since EA is closed under $\Sigma_{1}-\mathrm{CR}$, by Theorem 4.4 for $T=\mathrm{EA}$,

$$
\mathrm{EA}+\mathrm{Th}_{\mathscr{B}\left(\Sigma_{1}\right)}(S) \forall \mathrm{Th}_{\Pi_{2}}\left(\mathrm{EA}+\mathrm{Rfn}_{\Sigma_{2}}(\mathrm{EA})\right)
$$

and so $\operatorname{Th}_{\mathscr{B}\left(\Sigma_{1}\right)}(S) \forall \operatorname{Th}_{\Pi_{2}}\left(\operatorname{Th}_{\Sigma_{2}}(S)\right)$.

§5. Some results à la Kreisel-Lévy. In [13] Kreisel and Lévy showed that $P A$ is equivalent to the full uniform reflection principle over primitive recursive arithmetic. D. Leivant and $H$. Ono $[14,15]$ sharpened that result by showing that $\mathrm{EA}+\operatorname{RFN}_{\Sigma_{n+1}}(\mathrm{EA}) \equiv I \Sigma_{n}$ for each $n>0$. Later Beklemishev [2, 4] extended this correspondence to parameter free induction schemes and to theories described in terms of induction rules. In particular, he proved the following result over the base theory $\mathrm{EA}^{+}$(which is EA plus a $\Pi_{2}$-axiom declaring the totality of the superexponentiation function). $\mathrm{EA}^{+}$is needed because the proof uses formalized Cut-elimination Theorem. 
TheOREM 5.1. $[2,4]$ Suppose $T$ is a finite $\Pi_{2}$-extension of $\mathrm{EA}^{+}$.

1. $T+\mathrm{Rfn}_{\Sigma_{2}}(T) \equiv T+I \Pi_{1}^{-}$.

2. $T+\mathrm{Th}_{\Pi_{1}}(T+\operatorname{Rfn}(T)) \equiv T+\Pi_{1}-I R \equiv T+T_{\omega}$.

In the above theorem $T_{\omega}$ denotes the so-called $\omega$ times iterated consistency assertion for T, i.e., $\operatorname{Con}(T)+\operatorname{Con}(T+\operatorname{Con}(T))+\cdots$

In this section we improve Theorem 5.1 in two ways. On the one hand, we show that it also holds over the weaker base theory EA. On the other hand, we obtain a new Kreisel and Lévy-like result for $\operatorname{Rfn}_{\Sigma_{1}}(T)$.

We begin by showing that $\mathrm{EA}^{+}$can be dropped in part 1 in the above theorem thanks to a simple but useful trick. A similar result has been obtained by A. Visser (private communication).

THEOREM 5.2. If $T$ is a finite $\Pi_{2}$-extension of $\mathrm{EA}, T+\mathrm{Rf}_{\Sigma_{2}}(T) \equiv T+I \Pi_{1}^{-}$.

Proof. We only have to prove that $T+I \Pi_{1}^{-}$implies $\operatorname{Rfn}_{\Sigma_{2}}(T)$, as the opposite direction is already proved in [2] over EA. Suppose $\mathfrak{A} \models T+I \Pi_{1}^{-}$. It suffices to show that local $\Sigma_{2}$-reflection for Predicate Calculus PC is valid in $\mathfrak{A}$, for $T+\operatorname{Rfn}_{\Sigma_{2}}(T) \equiv T+\operatorname{Rfn}_{\Sigma_{2}}(\mathrm{PC})$ by the formalized Deduction theorem. Assume $\mathfrak{A} \models \exists y \operatorname{Prf} \operatorname{PC}_{\mathrm{PC}}(\ulcorner\varphi\urcorner, y)$. Since $\mathscr{K}_{1}(\mathfrak{A}) \prec_{1} \mathfrak{A}, \mathscr{K}_{1}(\mathfrak{A}) \models \exists y \operatorname{Prf} f_{\mathrm{PC}}(\ulcorner\varphi\urcorner, y)$. But it follows from $\mathfrak{A} \models \mathrm{EA}+I \Pi_{1}^{-}$and Proposition 4.1 that $\mathscr{K}_{1}(\mathfrak{A}) \models \mathrm{EA}^{+}$. Hence, Cutelimination is available and $\mathscr{K}_{1}(\mathfrak{A}) \models \exists y \operatorname{Prf}_{\mathrm{PC}}^{\mathrm{cf}}(\ulcorner\varphi\urcorner, y)$, where $\operatorname{Prf}_{\mathrm{PC}}^{\mathrm{cf}}(x, y)$ denotes cut-free provability. So, $\mathfrak{A} \models \exists y \operatorname{Prf}_{\mathrm{PC}}^{\mathrm{cf}}(\ulcorner\varphi\urcorner, y)$ as well and the result follows since the proof of Theorem 1 in [4] (see also Theorem 10 in [6]) shows that $I \Pi_{1}^{-}$implies local $\Sigma_{2}$-reflection for PC w.r.t. cut-free provability.

Now we turn to the promised theorem characterizing $\operatorname{Rfn}_{\Sigma_{1}}(T)$ by some form of induction. To this end, let $\Gamma$-IR and $\Gamma$-IR ${ }_{0}$ denote the inference rules

$$
\text { IR: } \frac{\varphi(0) \wedge \forall x(\varphi(x) \rightarrow \varphi(x+1))}{\forall x \varphi(x)} \quad \operatorname{IR}_{0}: \frac{\forall x(\varphi(x) \rightarrow \varphi(x+1))}{\varphi(0) \rightarrow \forall x \varphi(x)},
$$

where $\varphi(x) \in \Gamma$. In his detailed analysis of inference rules in arithmetic [2] Beklemishev proved that, if parameters are allowed, $\mathrm{IR}_{0}$ is congruent with the usual formulation of the induction rule: $\Sigma_{n}-\mathrm{IR}_{0} \cong \Pi_{n}-\mathrm{IR}_{0} \cong \Sigma_{n}$-IR. The parameter free version of $\mathrm{IR}_{0}$ was not considered there, however. It turns out that, whereas $\Sigma_{n}^{-}-\mathrm{IR}_{0}$ is easily seen to be congruent with $\Sigma_{n}-\mathrm{IR}_{0}, \Pi_{n}-\mathrm{IR}_{0}$ and $\Pi_{n}^{-}-\mathrm{IR}_{0}$ cease to be equivalent. In fact, we have

THEOREM 5.3. If $T$ is a finite $\Pi_{2}$-extension of $\mathrm{EA}$, then $T+\operatorname{Rfn}_{\Sigma_{1}}(T) \equiv T+$ $\Pi_{1}^{-}-I R_{0} \equiv\left[T, \Pi_{1}^{-}-I R_{0}\right]$.

Proof. Write $S$ for $T+\operatorname{Rfn}_{\Sigma_{1}}(T), R_{\omega}^{-}$for $T+\Pi_{1}^{-}-\mathrm{IR}_{0}$, and $R_{1}^{-}$for $\left[T, \Pi_{1}^{-}-\mathrm{IR}_{0}\right]$. First, observe that $R_{\omega}^{-}$is an extension of $T$ by $\mathscr{B}\left(\Sigma_{1}\right)$-sentences. Hence, $S \vdash R_{\omega}^{-}$ follows, since $T+\operatorname{Rfn}_{\Sigma_{2}}(T)$ implies $I \Pi_{1}^{-}$and is $\mathscr{B}\left(\Sigma_{1}\right)$-conservative over $S$. Second, the implication $R_{\omega}^{-} \vdash R_{1}^{-}$is trivial. Finally, we prove that $R_{1}^{-} \vdash S$. Suppose $\mathfrak{A} \models R_{1}^{-}$. It follows that $\mathscr{K}_{1}(\mathfrak{A}) \models\left[T, \Sigma_{1}-\mathrm{IR}\right]$. To see this, let $\varphi(x) \in \Sigma_{1}$ and assume $T \vdash \varphi(0) \wedge \forall x(\varphi(x) \rightarrow \varphi(x+1))$ and $\varphi(a)$ fails with $a \in \mathscr{K}_{1}(\mathfrak{A})$. Let $\delta(z)$ be a $\Sigma_{1}$-formula defining $a$. Applying $\Pi_{1}^{-}-\operatorname{IR}_{0}$ to $\forall z(\delta(z) \rightarrow \neg \varphi(z-x))$ we get a contradiction and the result follows. But $\left[T, \Sigma_{1}\right.$-IR] implies $T+\operatorname{RFN}_{\Sigma_{1}}(T)$ by Theorem 2 of [2]. Hence, $\mathfrak{A} \models T+\operatorname{Th}_{\Sigma_{2}}\left(T+\operatorname{RFN}_{\Sigma_{1}}(T)\right)$ and so $\mathfrak{A} \models S$. 
Now we show that $\mathrm{EA}^{+}$can also be dropped in part 2 of Theorem 5.1. This is interesting because in [2] Beklemishev remarked that, for characterizing $\Pi_{1}-$ IR by iterated consistency modulo EA one should replace $\operatorname{Con}(T)$ with a weaker notion of consistency based on cut-free provability. Nonetheless, Theorem 5.4 below will show that this result is true even for the usual notion of consistency.

THEOREM 5.4. If $T$ is a finite $\Pi_{2}$-extension of $\mathrm{EA}, T+\mathrm{Th}_{\Pi_{1}}(T+\operatorname{Rfn}(T)) \equiv$ $T+\Pi_{1}-I R \equiv T+T_{\omega}$.

PROof. Write $S$ for $T+\operatorname{Rfn}(T)$ and $R_{\omega}$ for $T+\Pi_{1}-$ IR. First, note that $T+$ $\mathrm{Th}_{\Pi_{1}}(S) \equiv T+T_{\omega}$ is proved over EA in Proposition 2.33 of [6] (a result that goes back to $\mathrm{S}$. V. Goryachev's [9]). Second, $T+\mathrm{Th}_{\Pi_{1}}(S) \vdash R_{\omega}$ because $R_{\omega}$ is an extension of $T$ by $\Pi_{1}$-sentences and $S \vdash I \Pi_{1}^{-}$by Theorem 5.2. Finally, we must prove that $R_{\omega} \vdash \operatorname{Th}_{\Pi_{1}}(S)$. By Theorem 1.1, $S$ is $\Pi_{1}$-conservative over $T+\operatorname{Rfn}_{\Sigma_{1}}(T)$ and so, by Theorem 5.3, it is sufficient to show that $\left[T, \Pi_{1}^{-}-\mathrm{IR}_{0}\right]$ is $\Pi_{1}$-conservative over $R_{\omega}$.

To this end, we use a model-theoretic method inspired by J. Avigad's [1] who, in turn, builds on some previous ideas of Visser (unpublished) and D. Zambella, [17]. The key notion is that of a $\Sigma_{1}$-closed model. We say that $\mathfrak{A}$ is $\Sigma_{1}$-closed with respect to (w.r.t.) a theory $U$ if $\mathfrak{A} \models U$ and, for every $\mathfrak{B} \models U, \mathfrak{A} \prec_{0} \mathfrak{B}$ implies $\mathfrak{A} \prec_{1} \mathfrak{B}$. By a union of chain argument every model of $U \subseteq \Pi_{2}$ can be $\Sigma_{0}$-elementarily extended to a $\Sigma_{1}$-closed model w.r.t. $U$. Thus, if every $\Sigma_{1}$-closed model w.r.t. $U \subseteq \Pi_{2}$ is a model of a theory $W$, then $W$ is $\Pi_{1}$-conservative over $U$ (see [7] for further details and applications of this method).

Turning back to the proof of the theorem, suppose $\mathfrak{A}$ is $\Sigma_{1}$-closed w.r.t. $R_{\omega}$. Assume $T \vdash \forall x(\varphi(x) \rightarrow \varphi(x+1))$, with $\varphi(x) \in \Pi_{1}^{-}$, and $\varphi(0)$ holds in $\mathfrak{A}$. It follows from the $\Sigma_{1}$-closedness condition that there is $\delta(a)$ in the $\Pi_{0}$-diagram of $\mathfrak{A}$ such that $R_{\omega} \vdash \delta(v) \rightarrow \varphi(0)$. Put $\varphi^{\prime}(x) \equiv \exists v \delta(v) \rightarrow \varphi(x)$. Clearly, $\varphi^{\prime}(x) \in \Pi_{1}^{-}$ and $R_{\omega}$ proves the antecedent of the induction axiom for $\varphi^{\prime}$. Hence, $\mathfrak{A} \models \forall x \varphi^{\prime}(x)$ and so $\mathfrak{A} \models \forall x \varphi(x)$ since $\mathfrak{A} \models \delta(a)$. Therefore, $\mathfrak{A} \models\left[T, \Pi_{1}^{-}-\mathrm{IR}_{0}\right]$.

§6. The case $n>0$. It is natural to ask ourselves how our results generalize to $\operatorname{Rfn}_{\Sigma_{n+2}}(T)$ and $I \Pi_{n+1}^{-}$for an arbitrary $n>0$. First of all, it should be noticed that, in order to characterize $I \Pi_{n+1}^{-}$in terms of reflection principles, one needs to consider relativized local reflection. For each $n>0$, the relativized local reflection principle for $T$ is the scheme given by

$$
\operatorname{Rfn}_{\Gamma}^{n}(T):[n]_{T}(\ulcorner\varphi\urcorner) \rightarrow \varphi,
$$

where $\varphi$ ranges over all sentences in $\Gamma$ and $[n]_{T}(x)$ denotes a $\Sigma_{n+1}$-formula expressing " $x$ is provable from $T+\operatorname{Th}_{\Pi_{n}}(\mathbb{N})$." That is $[n]_{T}(x) \equiv \square_{U}(x)$, where

$$
A x_{U}(x)=\left(A x_{T}(x) \vee \operatorname{True}_{\Pi_{n}}(x)\right)
$$

and $\operatorname{True}_{\Pi_{n}}(x)$ is a truth-definition for $\Pi_{n}$-sentences in EA (see Section 2.3 of [6] for details). It is a theorem of Beklemishev (see [4]) that over EA, $I \Pi_{n+1}^{-} \equiv \operatorname{Rfn}_{\Sigma_{n+2}}^{n}($ EA) and $I \Sigma_{n}^{-} \equiv \operatorname{Rfn}_{\Sigma_{n+1}}^{n}(\mathrm{EA})$ for each $n>0$. In addition, a relativization of Theorem 1.1 holds, i.e., $T+\operatorname{Rfn}_{\Sigma_{n+2}}^{n}(T)$ is $\mathscr{B}\left(\Sigma_{n+1}\right)$-conservative over $T+\operatorname{Rfn}_{\Sigma_{n+1}}^{n}(T)$. Thus, a relativized version of Question 1 is in order.

Question $3(n>0)$. Is $T+\operatorname{Rfn}_{\Sigma_{n+2}}^{n}(T)$ conservative over $T+\operatorname{Rfn}_{\Sigma_{n+1}}^{n}(T)$ with respect to $\Pi_{n+2}$-sentences? 
It turns out that the ideas in the previous sections apply equally well to the case $n>0$ for theories $T$ extending the $\Sigma_{n}$-induction scheme $I \Sigma_{n}$ and closed under $\Sigma_{n+1}$-collection rule. Such a theory $T$ can be reformulated as $I \Sigma_{n}+$ " $f$ is total", where $f$ is a nondecreasing function with a $\Pi_{n}$-graph. Thus, considering the $\Pi_{n+2}$ separation property $\forall x \in \mathscr{I}_{n+1} \forall z \in \mathscr{K}_{n+1}^{1}$ " $f^{x}(z)$ exists," allows us to obtain an analog of the unboundedness Theorem 4.4 for the class $\operatorname{Th}_{\Pi_{n+2}}\left(T+\operatorname{Rfn}_{\Sigma_{n+2}}^{n}(T)\right)$ and, in turn, a negative answer to Question 3.

It will be useful, however, to obtain an answer to Question 3 for theories $T$ extending EA rather than extending $I \Sigma_{n}$. To this end we have to use a different separation property. Now the key point is the following local variant of the finite axiom of choice for $\Pi_{n-1}$-formulas, denoted $F A C\left(\Pi_{n-1}, \mathscr{K}_{1}, \mathscr{K}_{n+1}^{1}\right)$,

$$
\forall v \in \mathscr{K}_{n+1}^{1} \forall z \in \mathscr{K}_{1}\left(\forall x \leq z \exists y \varphi(x, y, v) \rightarrow \exists s \forall x \leq z \varphi\left(x,(s)_{x}, v\right)\right),
$$

where $\varphi$ is in $\Pi_{n-1}$.

It is easy to check that $F A C\left(\Pi_{n-1}, \mathscr{K}_{1}, \mathscr{K}_{n+1}^{1}\right)$ can be reexpressed as a set of $\Pi_{n+2}$-sentences which are provable from $I \Pi_{n+1}^{-}$by Proposition 4.1 (and hence also from $\left.T+\operatorname{Rfn}_{\Sigma_{n+2}}^{n}(T)\right)$. By using this separation property we get:

THEOREM 6.1 ( $n>0$, Unboundedness). Suppose T is a $\mathscr{B}\left(\Sigma_{n+1}\right)$-extension of EA. Then, $\operatorname{Th}_{\Pi_{n+2}}\left(\operatorname{Rfn}_{\Sigma_{n+2}}^{n}(T)\right)$ is not contained in any consistent, r.e. extension of $T$ by $\mathscr{B}\left(\Sigma_{n+1}\right)$-sentences.

Proof. It is similar to that of Theorem 4.4 so we skip some details. Towards a contradiction, assume that there is an r.e. set of $\mathscr{B}\left(\Sigma_{n+1}\right)$-sentences $\Gamma$ such that $T+\Gamma$ is consistent and contains $\operatorname{Th}_{\Pi_{n+2}}\left(\operatorname{Rfn}_{\Sigma_{n+2}}^{n}(T)\right)$. In particular, $T+\Gamma+I \Sigma_{n}$ is consistent, for $I \Sigma_{n}$ is $\Sigma_{n+2}$-conservative over $I \Sigma_{n}^{-}$by Theorem 2.1 of [12]. Let $\mathfrak{A} \models T+\Gamma+I \Sigma_{n}$ with $\mathscr{K}_{1}(\mathfrak{A}) \neq \omega$ and $\mathscr{I}_{n+1}(\mathfrak{A}) \neq \mathfrak{A}$. Pick $a \in \mathscr{K}_{n+1}^{1}(\mathfrak{A})-\mathscr{I}_{n+1}(\mathfrak{A})$. By overspill inside $\mathscr{K}_{n+1}^{1}(\mathfrak{A})$ there is a sequence coded by $b \leq a$ such that,

$$
\mathscr{K}_{n+1}^{1}(\mathfrak{A}) \models \exists x, y \leq a \operatorname{Sat}_{n}(\ulcorner\theta\urcorner, x, y) \rightarrow(b)_{\ulcorner\theta\urcorner}=(\mu t)\left(\operatorname{Sat}_{n}\left(\ulcorner\theta\urcorner,(t)_{0},(t)_{1}\right)\right),
$$

for all $\theta(x, y) \in \Pi_{n}^{-}$, where $\mathrm{Sat}_{n}$ is a truth predicate for $\Pi_{n}$-formulas in EA and $(t)_{0},(t)_{1}$ denote the inverse of the Cantor pairing function. Note that $\mathscr{K}_{n+1}(\mathfrak{A})$ is included in any substructure of $\mathfrak{A}$ containing $b$, for in models of $I \Sigma_{n}$ every $\Sigma_{n+1}$-definable element can be obtained as the projection of a $\Pi_{n}$-minimal one. Since $b \in \mathscr{K}_{n+1}^{1}(\mathfrak{A})$, there are $b_{1} \in \mathscr{K}_{n+1}^{1}(\mathfrak{A})$ and $b_{2} \in \mathscr{I}_{n+1}(\mathfrak{A})$ satisfying that $b_{1}=(\mu t)\left(\varphi\left((t)_{0},(t)_{1}, b_{2}\right)\right)$ and $b=\left(b_{1}\right)_{0}$ for some $\varphi \in \Pi_{n}$. Put $c=\left\langle b_{1}, b_{2}\right\rangle$ and define $\mathfrak{B}$ to be $\mathscr{K}_{n}(\mathfrak{A}, c)$. Then $\mathfrak{B} \models T+\Gamma$ and $c \in \mathscr{K}_{n+1}^{1}(\mathfrak{B})$.

Finally $\mathfrak{B} \not \models F A C\left(\Pi_{n-1}, \mathscr{K}_{1}, \mathscr{K}_{n+1}^{1}\right)$. We argue as in Paris-Kirby' proof that the $\Sigma_{n}$-collection scheme fails in $\mathscr{K}_{n}(\mathfrak{A})$ (see Proposition 7 of [16]). Let $\operatorname{Min}_{n-1}(z, y, v)$ formalize " $z$ is the least element satisfying the $\Pi_{n-1}$-formula $y$ with a parameter $v$." If $\mathfrak{B}$ were to satisfy $F A C\left(\Pi_{n-1}, \mathscr{K}_{1}, \mathscr{K}_{n+1}^{1}\right)$ then, for any nonstandard $\Sigma_{1}$-definable element $d$, there would be an element $e$ such that

$$
\mathfrak{B} \models \forall x \leq d \exists y<d\left[\exists z \leq e\left(\operatorname{Min}_{n-1}(z, y, c) \wedge x=(z)_{0}\right)\right],
$$

violating the pigeon-hole principle for $\Sigma_{0}\left(\Sigma_{n-1}\right)$-functions in models of EA $+I \Sigma_{n-1}$. It follows that $T+\Gamma$ does not imply $F A C\left(\Pi_{n-1}, \mathscr{K}_{1}, \mathscr{K}_{n+1}^{1}\right)$, which contradicts our assumption that $T+\Gamma$ contains $\operatorname{Th}_{\Pi_{n+2}}\left(\operatorname{Rfn}_{\Sigma_{n+2}}^{n}(T)\right)$.

As a consequence, we obtain the corresponding negative answer to Question 3. 
COROLlaRY $6.2(n>0)$. Suppose $T$ is a $\mathscr{B}\left(\Sigma_{n+1}\right)$-extension of EA. $T+\operatorname{Rfn}_{\Sigma_{n+2}}^{n}(T)$ is not $\Pi_{n+2}$-conservative over $T+\operatorname{Rf}_{\Sigma_{n+1}}^{n}(T)$ provided that the latter theory is consistent.

In addition, taking $T=\mathrm{EA}$, Theorem 6.1 gives us that

COROLlARY $6.3(n>0) . I \Pi_{n+1}^{-}$is not $\Pi_{n+2}$-conservative over $I \Sigma_{n}^{-}$.

In [8] we gave another proof of Corollary 6.3 that uses a different separation property, namely, a formalized version of the model-theoretic property

$$
(A) \equiv \forall a \in \mathscr{K}_{n+1}^{1} \text { “ } \mathscr{K}_{n}(\mathfrak{A}, a) \text { is bounded above in } \mathfrak{A} . "
$$

This approach has the small advantage that it allows one to prove that $I \Pi_{n+1}^{-}$is not $\Pi_{n+2}$-conservative over $I \Sigma_{n}^{-}+B \Sigma_{n}$ for each $n>0$ (see the proof of Theorem 5.5 in [8] for details). Note that both $F A C\left(\Pi_{n-1}, \mathscr{K}_{1}, \mathscr{K}_{n+1}^{1}\right)$ and property $(A)$ above are provable from $I \Sigma_{n}$. This is no accident, as we shall infer from the following conservation result.

Let $\operatorname{Rfn}_{\Gamma}^{0}(T)$ coincide, by definition, with its non-relativized analog $\operatorname{Rfn}_{\Gamma}(T)$ and let $\operatorname{Rfn}^{n}(T)$ denote the full relativized local reflection principle for $T$.

THEOREM $6.4(n \geq 0)$.

1. $T+\operatorname{Rfn}^{n}(T)$ is $\Pi_{n+2}$-conservative over $T+\operatorname{RFN}_{\Sigma_{n+1}}(T)$.

2. $T+I \Pi_{n+1}^{-}$is $\Pi_{n+2}$-conservative over $\left[T, \Sigma_{n+1}-I R\right]$.

Proof. (1): Let $\varphi$ be a $\Pi_{n+2}$-sentence such that $T+\operatorname{Rfn}^{n}(T) \vdash \varphi$. Then, by a relativized version of Theorem 1.1, $T+\operatorname{Rfn}_{\Pi_{n+2}}^{n}(T) \vdash \varphi$. Using formalized Deduction theorem for $n>0$, we get $T+\operatorname{RFN}_{\Pi_{n+2}}(T) \vdash \varphi$. Finally, note that over $\mathrm{EA}, \operatorname{RFN}_{\Pi_{n+2}}(T) \equiv \operatorname{RFN}_{\Sigma_{n+1}}(T)$ and hence the result follows.

(2): Let $\varphi$ be a $\Pi_{n+2}$-sentence provable in $T+I \Pi_{n+1}^{-}$. Since $I \Pi_{n+1}^{-} \subseteq \Sigma_{n+2}$, by compactness there is a finite $\Pi_{n+2}$-axiomatized subtheory $T_{0} \subseteq T$ satisfying that $T_{0}+I \Pi_{n+1}^{-} \vdash \varphi$. Then, by a relativized version of Theorem 1.1, $T_{0}+\operatorname{Rfn}_{\Sigma_{n+2}}^{n}\left(T_{0}\right) \vdash \varphi$. Then, trivially, $T_{0}+\operatorname{Rfn}^{n}\left(T_{0}\right) \vdash \varphi$ and, by part (1), $T_{0}+\operatorname{RFN}_{\Sigma_{n+1}}\left(T_{0}\right) \vdash \varphi$. But $\left[T_{0}, \Sigma_{n+1}\right.$-IR] $\equiv T_{0}+\operatorname{RFN}_{\Sigma_{n+1}}\left(T_{0}\right)$ by Theorem 4 of [2] and the result follows. -1

By using a model-theoretic construction Kaye, Paris and Dimitracopoulos proved that, when formulated in the usual language of arithmetic, $I \Pi_{1}^{-}$is $\Pi_{2}$-conservative over $I \Delta_{0}+\exp \equiv\left[I \Delta_{0}, \Sigma_{1}\right.$-IR] (see Theorem 2.9 of [12]). Thus, Theorem 6.4 can be seen as a reflection principle counterpart of Kaye-Paris-Dimitracopoulos' result. Since $\left[\mathrm{EA}, \Sigma_{n+1}-\mathrm{IR}\right] \equiv \mathrm{EA}+\mathrm{RFN}_{\Sigma_{n+1}}(\mathrm{EA}) \equiv I \Sigma_{n}$, taking $T=\mathrm{EA}$ in Theorem 6.4 yields the following new conservation result for induction schemes.

Corollary $6.5(n>0) . I \Pi_{n+1}^{-}$is $\Pi_{n+2}$-conservative over $I \Sigma_{n}$.

Corollary 6.5 explains why any $\Pi_{n+2}$-property separating $I \Pi_{n+1}^{-}$and $I \Sigma_{n}^{-}$turns out to be provable from $I \Sigma_{n}$ as well as, combined with Corollary 6.3 , settles the question of the optimality of conservativity between $I \Pi_{n+1}^{-}$and $I \Sigma_{n}^{-}$for $n>0$. On the one hand, $I \Pi_{n+1}^{-}$is $\mathscr{B}\left(\Sigma_{n+1}\right)$-conservative over $I \Sigma_{n}^{-}$and this result is best possible; on the other hand, conservativity of $I \Pi_{n+1}^{-}$over $I \Sigma_{n}$ can be extended to $\Pi_{n+2}$-sentences and this is, again, best possible.

Acknowledgements. The authors are grateful to the anonymous referees for their helpful suggestions for improving the presentation of the paper. 


\section{REFERENCES}

[1] J. Avigad, Saturated models of universal theories, Annals of Pure and Applied Logic, vol. 118 (2002), pp. 219-234.

[2] L. D. BEKLEMISHEv, Induction rules, reflection principles, and provably recursive functions, Annals of Pure and Applied Logic, vol. 85 (1997), pp. 193-242.

[3] - Notes on local reflection principles, Theoria, vol. 63 (1997), pp. 139-146.

[4] - Parameter free induction and provably total computable functions, Theoretical Computer Science, vol. 224 (1999), pp. 13-33.

[5] — Proof-theoretic analysis by iterated reflection, Archive for Mathematical Logic, vol. 42 (2005), pp. 515-552.

[6] - Reflection principles and provability algebras in formal arithmetic, Russian Mathematical Surveys, vol. 60 (2005), pp. 197-268.

[7] A. Cordón-Franco, A. Fernández-Margarit, and F. F. LaRa-Martín, On conservation results for parameter-free $\pi_{n}$-induction, Studies in weak arithmetics (P. Cégielski, editor), CSLI Lecture Notes, vol. 196, The University of Chicago Press, 2010, pp. 49-97.

[8] - A note on parameter free $\pi_{1}$-induction and restricted exponentiation, Mathematical Logic Quarterly, vol. 57 (2011), pp. 444-455.

[9] S. V. GORYACHEv, On the interpretability of some extensions of arithmetic, Rossiüskaya Akademiya Nauk. Matematicheskie Zametki, vol. 40 (1986), pp. 561-571.

[10] P. HÁJEK and P. PUDLÁ, Metamathematics of first-order arithmetic, Perpectives in Mathematical Logic, Springer-Verlag, 1993.

[11] R. KaYe, Models of Peano Arithmetic, Oxford Logic Guides, vol. 15, Oxford University Press, 1991.

[12] R. KaYe, J. Paris, and C. Dimitracopoulos, On parameter free induction schemas, this Journal, vol. 53 (1988), pp. 1082-1097.

[13] H. KREISEL and A. LEVY, Reflection principles and their use for establishing the complexity of axiomatic systems, Zeitschrift für mathematische Logik und Grundlagen der Mathematik, vol. 14 (1968), pp. 97-142.

[14] D. LEIVANT, The optimality of induction as an axiomatization of arithmetic, this JouRNAL, vol. 48 (1983), pp. 182-184.

[15] H. ONO, Reflection principles in fragments of peano arithmetic, Zeitschrift für mathematische Logik und Grundlagen der Mathematik, vol. 33 (1987), pp. 317-333.

[16] J. PARIS and L. KIRBY, $\Sigma_{n}$-collection schemas in arithmetic, Logic colloquium 77 (A. Macintyre, L. Pacholski, and J. Paris, editors), North-Holland, 1978, pp. 285-296. 966

[17] D. ZAMBella, Notes on polynomial bounded arithmetic, this JouRNAL, vol. 61 (1996), pp. 942

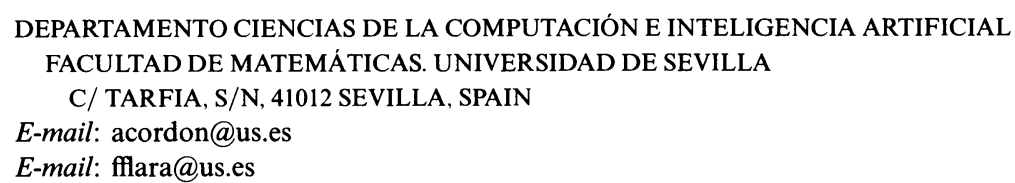

